\title{
Diversidad funcional e inclusión en Instituciones de Educación Superior (IES) en Medellín
}

\author{
Functional diversity and inclusion in Institutions of Higher Education (IES) \\ in Medellín
}

Sandra Isabel Mejía Zapata

Psicóloga. Especialista en docencia investigativa universitaria, Magíster en intervenciones psicosociales. Docente investigadora en la Facultad de Psicología y Ciencias Sociales de la Universidad Católica Luis Amigó, Medellín, Colombia. E-mail: sandra.mejiaza@amigo.edu.co

“Cada persona brilla con luz propia entre todas las demás. No hay dos fuegos iguales. Hay fuegos grandes y fuegos chicos y fuegos de todos los colores."

Eduardo Galeano

\section{Resumen}

El trabajo que se informa tuvo como objetivo presentar la realidad evidenciada en materia de educación superior inclusiva para las personas con diversidad funcional (discapacidad) en Medellín, Colombia. Para ello, se implementó una investigación cualitativa, teniendo como instrumento una entrevista en profundidad que constaba de 25 preguntas que apuntaban a esclarecer las posibilidades de acceso a la educación, las condiciones para la permanencia y el reconocimiento de los apoyos (ajustes razonables) existentes y requeridos a partir de la visión de los distintos actores educativos. La entrevista fue administrada a dos estudiantes universitarios con diversidad funcional, un docente de educación superior y un funcionario de bienestar universitario por cada una de las nueve universidades seleccionadas en el área metropolitana de Medellín; la información obtenida se sistematizó en el software Atlas.ti y posteriormente se trianguló para su análisis, permitiendo la emer- gencia de las categorías finales. Se identificó que en la mayoría de las universidades no se tiene acceso a espacios de uso común tales como bibliotecas y auditorios. Solo en una de las nueve IES se registró la existencia de una política interna que aseguraba las condiciones para la educación inclusiva de la población con diversidad funcional. Lo anterior exhorta a revisar y trascender los enfoques que actualmente rigen el diseño de la política pública de la discapacidad en aras de garantizar el derecho constitucional a una educación con calidad que dignifique y permita la participación plena de este colectivo.

Palabras clave: Diversidad funcional; Educación superior; Derechos; Política pública y Participación.

\section{Abstract}

The main objective of this article is to present the reality evidenced in the field of higher education and the inclusive policies for 
people with functional diversities (disability) in the city of Medellin, Colombia. According to this, a qualitative research was implemented that allowed to recognize the perceptions and experience in this population. For this, a deep interview was used, with 25 questions that were focused on three main topics: access to educative opportunities, the conditions for permanence and the recognition of the support given (reasonable accommodations), according to the points of view of different educational actors. This same interview was applied to three different population groups. The first one was formed by 18 university students with functional diversities, between the ages of 22 and 30 years old. The second group was composed of 16 undergraduate students, and two from the postgraduate courses. In addition, they were classified by their functional diversity: ten of them had physical or motor disabilities (two didn't have upper limbs, two had muscular dystrophy and six had reduced mobility; in this group, four of them were born with those disabilities and two acquired them during their life). The other group had sensorial disabilities (two had deafness, three were blind and three had reduced vision). The third group was composed of 9 university teachers and 9 staff members of the university welfare department. All of them live in Medellin.

The information was systematized in the Atlas.ti software and then triangulated. A comparative matrix was elaborated according to the associated principles of continuity, similarity and contrasting significant relations for the analysis and generating the appearance of final categories. In this regard, it is recognized that inclusive education merits universities as the entities which assume places full of transformation. This situation isn't visualized in this investigation because only one out of nine have been complying. Three of them are in the implementation process and the others assume that inclusive education is where the student has to adapt to the institutional conditions.

It was identified that the admission process didn't take into account equitable conditions.
Thus, it would be understanding that the characteristics are comparable at the diverse population, taking into account their needs and abilities. In general, universities don't provide handicap access to some common places like libraries and auditorium. Functional diversity support is mainly implemented for the population who has reduced mobility; this implies structural adjustments like ramps and elevators, among other possibilities. Only in one out of nine universities there was evidence of the existence of an internal policy put in place to guarantee the conditions needed for people with functional diversity.

It is possible that one of the most evident situations has to do with the teacher's commitment, an aspect that has a considerable impact in the inclusive education. However, in spite of good wills, this question needs go further. It requires skills and training to recognize the support a particular diversity or handicap requires. Most of them expressed concern and anxiety for their ignorance about the matter; that many cases make the reference population invisible. Within the range of competences, it has to develop some skills that include technological, methodological and curricular skills. But also, there are other soft competences that designate creativity, respect, compromise, and tolerance, among others. The educational integration implies a joint effort form all the educational community. Furthermore, the human factor promotes the adaptation process significantly in the educational environment. Meanwhile, they are configured as social supports, in opposition to the barriers established by limiting the participation and recognition of the same. In light of all this, it is important to review and go further than the approaches that are currently applied in the design of the public policies focused in disabilities, to guarantee the constitutional right to a quality education and to also dignify this group and provide them with full participation.

Keywords: Functional diversity; Higher education; Rights; Public policies; Participation. 


\section{Introducción}

Los derechos para las personas con diversidad funcional en el contexto colombiano aún se reconocen bajo el modelo médico rehabilitador.

"La diversidad se vincula al antiguo modelo médico que dio inicio a la temática de la integración en los años 70's, que identifica a estos sujetos como aquellos que poseen limitaciones físicas y/o cognitivas que dificultan el proceso de aprendizaje en el aula, enfatizando en las dificultades y responsabilidades que ellos poseen para 'adaptarse' a la sociedad, desconociendo la responsabilidad del entorno para colaborar en dicho proceso adaptativo" (Rubio, 2017, p. 209).

En educación superior, las oportunidades son reducidas en tanto las instituciones de educación superior (IES) no están adaptadas para acompañar una propuesta educativa integral y equitativa. La inexistencia de una política interna implica que siga siendo el estudiante quien debe adaptarse a la realidad institucional y su admisión relegada a mínimas condiciones, dando lugar al incremento de la deserción educativa $\mathrm{y}$, por obvias razones, al debilitamiento del proyecto de vida de las personas con diversidad funcional.

La presente investigación busca promover condiciones dignas para las personas con diversidad funcional a partir del reconocimiento de los apoyos y la eliminación de las barreras que dificultan las oportunidades y la participación. Asimismo, pretende provocar en la población materia de investigación una postura política y emancipadora en tanto aboguen por sus derechos, dando lugar a la autodeterminación.

Colombia se sitúa aún en el modelo social propuesto en la Convención sobre los derechos de las personas con discapacidad (CRPD, por sus siglas en inglés). Sin embargo, se adoptará el modelo de la diversidad funcional, en tanto permite una apuesta diferenciadora que pone el énfasis básicamente en dos aspectos: el primero, la promoción de un sujeto con autodeterminación, es decir, que puede tomar decisiones sobre su propia vida; y el segundo aspecto radica en el logro de la dignidad plena para la población en mención.

Respecto a la dignidad: "Es inherente a todos los seres humanos y no está vinculada a la capacidad. Es en este concepto de dignidad en el que parece estar una de las claves de las incoherencias discriminatorias de la realidad actual" (Palacios y Romañach, 2008, p. 41). La asociación entre capacidades y funcionamientos (biología) determina sustancialmente la valía de las personas en esta condición, supeditando la dignidad a un asunto que se otorga.

El modelo de la diversidad funcional surge en el año 2005 en España, propuesto por el colectivo de vida independiente, conformado por un grupo de personas en esta condición, quienes estimaban conveniente generar un impacto en el diseño de la política pública que favorece a la población, bajo el lema "Nada para nosotros sin nosotros", acuñado por el Foro de Vida Independiente, en 2005. Es determinante que la política en educación superior sea pensada y ajustada desde la perspectiva de la población con diversidad funcional (Valenzuela-Zambrano, Panão Ramalho, Chacón y López, 2017).

En Colombia, la educación superior para las personas con diversidad funcional es concebida por terceros que trazan una línea desde la inclusión educativa, la cual difiere en sus concepciones de la educación inclusiva; la primera exige que el estudiante con diversidad funcional se adapte a las condiciones institucionales, mientras que la segunda se prepara para posibilitar los apoyos necesarios en una educación equitativa a partir del diseño de políticas internas, planes y proyectos que redimensionen la permanencia educativa para este colectivo (Velásquez, 2009).

La revisión parte desde los procesos de selección para ellas, en tanto las pruebas en la mayoría de las IES son estandarizadas y requieren de ajustes que "para el caso serían solo en la forma en cómo se presenta, porque 
el cambiar contenidos y extensión implica el debilitamiento en la validez del instrumento" (Chiny Naranjo, Salas Pérez y Vargas Dengo, 2008, p. 78). Esta se concibe como una razón de peso por la cual las instituciones no realizan ajustes, pero además muy pocas cuentan con pruebas específicas de aplicación para la población con diversidad funcional; en muchos de los casos ni siquiera tienen conocimiento acerca de qué sería pertinente evaluar en ellas.

Lo expresado anteriormente impacta significativamente en el acceso a la educación superior para este colectivo, teniendo en cuenta que, según la Organización Mundial de la Salud (OMS), en la actualidad el $15 \%$ de la población mundial está en condición de diversidad funcional y el $6.3 \%$ está en Colombia, el segundo país del mundo con mayor población en esta condición; tan solo en el departamento de Antioquia hasta 2015 se reportaron 387313 personas, de las cuales aproximadamente 15640 están en Medellín. Entrando en materia de educación superior, 238 de ellas acceden a educación técnica o tecnológica, 148 a la universidad, de las cuales 32 obtienen el título y únicamente 6 personas logran realizar un posgrado (Departamento Administrativo Nacional de Estadística, 2015).

Esta es una de las tantas barreras que enfrentan, ya que "por encima del cumplimiento y eficacia de las políticas, se observa cierta negligencia institucional para la implementación de una educación inclusiva" (Ricardo, 2012, p. 50) dando lugar a barreras de tipo psicosocial. Al respecto, "se hace determinante suprimir las trabas que impidan la participación de las personas con diversidad funcional; de la misma forma, se requiere instruir a la comunidad académica y posibilitar las adecuaciones respectivas conforme a las necesidades de los estudiantes" (Fernández de Álava, Barrera Corominas y Díaz Vicario, 2013, p. 111). Además,

"algunos aspectos de tipo académico se configuran como problemas en sí para la permanencia de los estudiantes con diversidad funcional en las IES, tales como el exceso de créditos académicos, insuficientes estrategias didácticas y tecnológicas, que en muchos casos dificultan el cumplimiento de los eventos evaluativos, generando ansiedad significativa, más aún que en otros niveles de educación en la etapa escolar, donde las estrategias pedagógicas suelen diversificarse" (Alarcón, Lissi, Medrano y Loret, 2013, p. 89).

"La educación inclusiva se ve en su mayoría afectada por barreras psicosociales (administrativas, económicas y actitudinales) que se consolidan como impedimentos limitando las oportunidades, la participación y por ende una educación integral y equitativa" (Ricardo y Angarita, 2014, p. 95).

Otro aspecto relevante radica en que la realidad institucional para cada IES es multidimensional e implica la revisión particular; además, la implementación de la norma es variable. "Los lineamientos emitidos por el Ministerio de Educación Nacional no son acatados en su totalidad, en virtud de que las IES tienen autonomía universitaria y no están obligados a aceptar una fuerza política que debe ser ejecutada" (Ricardo y Angarita, 2014, p. 91).

Es necesario, bajo la realidad evidenciada, "establecer una política clara que favorezca la inclusión, que contemple planes y programas como: establecimiento de estrategias pedagógicas orientadas a la atención de las NEE, capacitación docente en el uso de adaptaciones curriculares, desarrollo de programas culturales y deportivos que coadyuven en la formación integral de los estudiantes con discapacidad, todo lo anterior unido a estrategias de comunicación y sensibilización que permitan la formación o el fortalecimiento de una comunidad universitaria que acoja a las personas en situación de discapacidad sin discriminación alguna" (Fernández y Duarte, 2016, p. 101).

Los antecedentes planteados permiten reconocer que la situación es de grandes proporciones, ya que si bien existe la política pública que aboga por las condiciones de las personas 
con diversidad funcional, se cumple en las IES en la mínima proporción para evitar las sanciones, lo cual es insuficiente para suplir las necesidades de esta población y más aún para garantizar el derecho a una educación inclusiva. Aunque existen algunas IES que hacen un esfuerzo considerable por convertirse en entornos accesibles e incluyentes, son la minoría.

Se pretende identificar la realidad contextual en materia de educación superior y diversidad funcional desde tres perspectivas fundamentales: las áreas de bienestar universitario, los docentes de los diversos programas académicos y la población con diversidad funcional en las IES.

\section{Métodos}

Se realizó una investigación cualitativa de corte fenomenológico, en tanto permitió una explicación desde la experiencia subjetiva de las personas (Galeano, 2003) implementando la modalidad de historias de vida.

Para ello se diseñó como estrategia metodológica una entrevista en profundidad, que facilitó determinar criterios para el acceso y permanencia educativa para estas personas desde las unidades de bienestar universitario de nueve instituciones de educación superior (IES), las percepciones de la población en cuestión y por último el sentir de los docentes frente al acto educativo. Su ejecución data de febrero de 2015 a enero de 2016. Los datos utilizados son parte de un banco de datos recopilados durante la maestría de la autora y su tarea como asesora de trabajos de grado del pregrado en Psicología de la Universidad Católica Luis Amigó (Funlam, Medellín, Colombia).

\section{Participantes}

En el presente estudio se trabajó en nueve IES del área metropolitana de Medellín, teniendo en cuenta como criterios de inclu- sión, la representatividad de las mismas en la región. Desde su naturaleza jurídica, tres eran públicas y seis privadas; en cuanto a los niveles de estratificación se identifican seis IES con el nivel bajo medio y tres con nivel medio alto. En todas se contaba con población con diversidad funcional motora y sensorial.

Con respecto a la selección de informantes, la muestra se efectuó mediante un diseño secuencial, de tipo bola de nieve, en la cual "se va decidiendo sobre la marcha, es donde se identifican los casos de interés a partir de alguien que conozca a alguien que puede resultar un buen candidato para participar" (Martínez-Salgado, 2012, p. 616). Asimismo "la muestra pretende criterios de comprensión y pertinencia no de representatividad estadística" (Galeano, 2003, p. 33). Por cada institución se eligió a dos informantes con diversidad funcional (sensorial o motora), mayores de edad, un docente y un administrativo de bienestar universitario. Se excluyeron del estudio personas con diversidad funcional cognitiva y psicológica (autismo, Down, entre otras).

Se entrevistó a 18 estudiantes con diversidad funcional en condición de adultez temprana (Papalia, Wendkos y Duskin, 2001), para el caso entre los 22 a 30 años. Teniendo en cuenta el nivel académico, 16 eran de pregrado y 2 de posgrado; en cuanto a la tipología de la diversidad funcional (World Health Organization, en la Clasificación Internacional del Funcionamiento de la Discapacidad y la Salud-CIF, 2001), 10 eran de tipo motora ( 2 con ausencia de miembros superiores, 2 con distrofia muscular y 6 con movilidad reducida -4 de nacimiento y 2 adquirida-) y 8 de tipo sensorial ( 2 con hipoacusia, 3 ciegos y 3 con baja visión).

Para los nueve docentes se tuvo en cuenta que además hubiesen tenido experiencias con estudiantes en condición de diversidad funcional. Respecto a los funcionarios de bienestar universitario se entrevistaron nueve, uno por cada institución, que estuviesen a cargo de lo concerniente a la población. 


\section{Instrumentos}

Se aplicó una entrevista en profundidad, subrayando que posibilita conocer de manera amplia y sin sesgos las vivencias de las personas entrevistadas (Taylor y Bogdan, 1992). La misma constaba de 25 preguntas abiertas, repartidas entre los segmentos poblacionales: a) 18 estudiantes con diversidad funcional (11 ítems) agrupadas en dos tópicos: el ingreso a la educación superior, la permanencia y los apoyos requeridos durante la etapa de la educación superior; b) nueve docentes ( 8 ítems) desde dos tópicos: vivencia de la docencia con la población específica y reconocimiento de la política institucional; c) nueve funcionarios de bienestar universitario (6 ítems) en un tópico: la realidad institucional en materia de educación inclusiva para la población con diversidad funcional.

La entrevista fue sometida a pilotaje por pares, permitiendo la validación y los ajustes requeridos de acuerdo a las pretensiones que se tenía con cada segmento poblacional. Cada una fue grabada y posteriormente transcripta para sistematizarla en el software Atlas.Ti.

\section{Procedimiento}

La investigación presenta unas fases que si bien muestran un proceso organizado no guardan estrictamente una linealidad en tanto el enfoque cualitativo no es reductible a etapas. Consiste en una interacción que permite la construcción simultánea entre las mismas (Galeano, 2003). Se hablará entonces de momentos en el proceso.

Momento de exploración: se delimitó la temática y los objetivos del estudio, seguidamente se realizó el rastreo de antecedentes de punta y teóricos, estableciendo que fueran de carácter científico y estuvieran consignados en bases de datos académicas; de la revisión derivó la pregunta de investigación: “¿cómo es el panorama actual en Medellín, en materia de educación inclusiva, en las IES?".
Se realizó la codificación (Hernández Sampieri, Fernández Collado y Baptista Lucio, 2010) de los antecedentes en el software Atlas Ti, asignando palabras clave; luego los códigos se sintetizaron por semejanza, agrupándolos en categorías iniciales de análisis, dando lugar a las categorías descriptivas: diversidad funcional, políticas para la diversidad funcional, derechos, educación inclusiva, instituciones de educación superior, docencia para la diversidad funcional.

Se diseñó el protocolo de la entrevista bajo los tópicos mencionados en la descripción del instrumento, se la sometió a revisión por pares expertos en el tema para asentir su pertinencia, se elaboró el protocolo de consentimiento informado (FO-MI-153, versión 1 - 2-12-2011), el cual fue socializado con los entrevistados. En ese protocolo se delimitó el alcance del estudio y las implicaciones de participar; además se implementó un sistema de codificación tanto para informantes como para las instituciones, en aras de la discrecionalidad y confidencialidad de los datos posteriores a su aplicación. Se realizaron las entrevistas a profundidad y fueron categorizadas para el análisis de la información. Con respecto a la codificación implementada, se asignó a cada una de las nueve instituciones una letra mayúscula desde la A hasta la I, que corresponde a la primera convención utilizada en la nomenclatura; para los docentes se utilizó la letra D; para Bienestar Universitario la letra B y para los estudiantes con diversidad funcional, E1 y E2, respectivamente.

Momento de focalización: Se realizó una matriz comparativa teniendo como parámetro los principios asociacionistas de contraste, semejanza y contigüidad (Del Carmen, 2009) posibilitando la triangulación entre antecedentes teóricos, de punta y las entrevistas generando categorías explicativas.

Momento de profundización: permite el reconocimiento del estado de la educación inclusiva para las personas con diversidad funcional a la luz de las categorías interpretativas: educación superior y diver- 
sidad funcional, docencia para la diversidad funcional, del desconocimiento al compromiso y educación inclusiva, un reto a alcanzar.

\section{Resultados}

Los resultados evidencian las categorías emergentes del proceso, analizadas a partir de las narrativas de los informantes, permitiendo así la caracterización del estado de la educación superior inclusiva en Medellín, Colombia.

\section{Educación superior y diversidad funcional}

Esta categoría permite reconocer el sentir y las vivencias desde la población con diversidad funcional, con respecto a las condiciones y características para el ingreso educativo teniendo en cuenta los procesos de selección de aspirantes. Puede identificarse que en la mayoría de las IES dichos procedimientos se encuentran estandarizados, sin diferenciar su aplicación de las personas convencionales (sin diversidad funcional).

El protocolo se compone generalmente de una entrevista grupal, que en el caso de la diversidad funcional motora puede requerir cambio de espacios en tanto se imposibilita el acceso, generando inconvenientes de última hora.

"Cuando llegué a la entrevista me descorazoné, no voy a poder entrar aquí dije, la entrevista era en el tercer piso y yo en esta silla, pero afortunadamente la persona que la realizaba bajó a la cafetería y allí fue, ahora sigue siendo difícil pero mis compañeros me cargan hasta arriba" (F.E.2, 2017).

En el caso anterior podría pensarse que un cambio de sitio es un contratiempo menor, sin embargo, el asumir por cuenta del estudiante, el ser trasladado como lo refiere, pone en riesgo su integridad física y no da cuenta de condiciones equitativas y dignificantes.

Para el caso de la diversidad funcional sensorial, se requiere de apoyos significativos que implican recursos tecnológicos, humanos y materiales, si se tiene en cuenta que muchas personas sordas no están oralizadas y deben tener un intérprete en lengua de señas. De la misma manera las personas ciegas o con baja visión estiman del apoyo del braille o de software como el magic o el jaws, que cumplen la función de pasar el texto a audio, lo cual es determinante para la segunda parte del proceso, que consiste en la aplicación de pruebas psicotécnicas que tienen como función identificar algunas competencias personales para cumplir con el perfil de cada profesión.

"Fue complicado sí, me tocó aplazar la entrevista, ya que no tenían intérprete y paila, yo dije 'qué va, ya no me llaman', pero sí, casi a punto de entrar sonó el celular y me dijeron que podía ir" (A.E.1, 2016).

"Cuando entré y me entregaron las hojas de los test, yo dije "es que no las puedo hacer porque soy ciega', entonces la niña me dijo que tenía que esperar a ver cuál era el procedimiento" (G.E.2, 2016).

Tres de las IES manifestaron tener en la plataforma de inscripción para los aspirantes, un campo que permite la opción de identificarse como persona con diversidad funcional, lo que posibilita realizar la previsión de los apoyos que se requieren para la valoración y admisión del estudiante; sin embargo, solo unos pocos lo hacen por temor a la identificación y rechazo por su condición, en tanto requiere de apoyos para la permanencia.

"Yo sí vi ese espacio, pero ya me han rechazado de una universidad, nunca me llamaron y debe haber sido por eso, así que no, mejor no lo puse, después creen que uno es una carga, pero aquí estoy y aquí me quedé, hay que tener viveza" (I.E.2, 2016).

Es transversal en 12 de los 18 estudiantes entrevistados que no se sentían cómodos, en los primeros acercamientos con la IES para develar su condición, ya que en ocasiones habían sido excluidos por su diversidad funcional, lo que dificulta significativamente el optimizar las estrategias que para la educación inclusiva puedan tenerse en las instituciones.

Otro de los elementos materia de revisión con la población al interior de las IES, consiste 
en reconocer cómo se promueve la permanencia, entendida como el cumplimento de las condiciones institucionales que permitan al estudiante con diversidad funcional un entorno adaptable, accesible y participativo, que no será posible sin los apoyos específicos desde cada una de las particularidades de los estudiantes en esta condición.

Al respecto la población en tres de las instituciones, dos de naturaleza privada (IES B y G) una de carácter público (IES F), todas de estrato bajo medio, presentan escaso o ningún ajuste, dando cuenta de que los derechos de los estudiantes con diversidad funcional quedan relegados a la inclusión educativa, donde son ellos los que deben adaptarse a las instituciones.

"En mi caso solo está la rampa de la entrada, aunque es muy incómoda, un día se me volteó la silla y casi me rompo la cabeza, porque es muy estrecha, pero adentro me cargan mis compañeros o el vigilante, ya que la institución es de tres plantas y no hay ascensor" (F.E.2, 2016).

"La universidad tiene cinco bloques, en uno de ellos hay ascensor y se ubican dos rampas, una a la entrada del edificio y otra al interior, a mí la verdad no me va tan mal, no sé qué pasará con algunos estudiantes que distingo que son ciegos y hasta un sordo, para ellos es más difícil" (B.E.2, 2016).

En dos universidades, una pública de estrato medio bajo (IES.E) y otra privada de estrato alto (IES.I), los estudiantes manifestaron los mayores desarrollos a nivel infraestructural y tecnológico; en (IES.E) incluso se contaba con un programa de formación profesional exclusivo para personas sordas, implementando lo requerido para que accedieran a una educación de calidad. En la otra institución (IES.I), no escatimaban en el tema de adecuaciones locativas y curriculares para posibilitar la participación y el cumplimiento de derechos de la población en cuestión.

"Yo soy una persona en condición de discapacidad sensorial-auditiva, hay un esfuerzo muy positivo para el ingreso de las personas sordas, se cuenta con lo que necesitamos, yo estoy muy contento con el apoyo que me dan y la forma en que acompañan los profes" (E.E.1, 2016).

"En mi caso que estoy en silla de ruedas, me colaboran con el cambio de aula cuando es en un segundo o tercer piso, donde uno no puede tener el acceso, hay rampas por todas partes, hay parqueaderos para personas con movilidad reducida, los baños están acondicionados con todas las cosas para la gente que tiene una diversidad, la universidad sí cuenta con todo para el buen acceso" (I.E.1).

En las instituciones restantes (IES.A, pública, e IES.C, D y H), se reconoció en la materia, un desarrollo medio en el tema de la educación inclusiva, en tanto se identificaron adecuaciones locativas sustanciales, algunas realizadas bajo la demanda apremiante, que en ciertos casos no cumplen con las especificaciones técnicas; se reconocen adecuaciones de tipo curricular mínimas y se cuenta con software y tecnología en pequeña escala, pero que permitía los apoyos en cuestión. Un estudiante ciego refiere: "En el caso de materias que son un poco visuales como la psicobiología, la docente hizo modificaciones curriculares, imagínense que hizo una neurona en plastilina para que pudiera ver cómo es cada parte de la neurona y pues sus divisiones y diferentes estructuras" (H.E.1, 2016).

Uno de los aspectos a señalar es que, de la población entrevistada la más vulnerable y desprotegida es la población sorda, que no está oralizada, ya que se requiere de la contratación del intérprete incrementando significativamente el costo en el tema de los apoyos, además de requerirla adecuación a profundidad en la malla curricular, en el caso de una segunda lengua, para muchos de ellos.

\section{Docencia para la diversidad funcional, del desconocimiento al compromiso}

Otro asunto relevante para la profesionalización de las personas con diversidad funcional, es la docencia como un factor 
contundente que incide en la calidad de la educación y en las oportunidades para la equidad y la participación, en tanto su rol amerita reconocer tres elementos. El primero de ellos es la necesidad de realizar ajustes metodológicos y curriculares, para los cuales muchos docentes no están preparados. A propósito, sobre la pregunta si se sentían preparados para el acompañamiento con la población, pudo identificarse lo siguiente:

"No, trabajar con personas con discapacidad requiere de cierta capacitación dependiendo de la discapacidad de las personas, estamos trabajando con personal con alguna deficiencia por ejemplo auditiva, necesitamos saber un lenguaje de señas para poder comunicarnos con ellos, en este caso nosotros no estamos preparados para eso" (E.D.1, 2016).

"No, porque me parece que es algo complejo, no tengo los elementos suficientes, desconozco muchos elementos de información, desde la investigación, de lo que realmente sucede en algunos casos muy puntuales para asumir en el aula de clase y poder enfrentarlo de una manera adecuada" (C.D.1, 2016).

En segunda instancia los tiempos asignados son para dedicación exclusiva a actividades que fortalezcan las funciones sustantivas (docencia, extensión, investigación, internacionalización y bienestar universitario) y por último está la voluntad del docente, que si bien en algunos casos se compromete, en otros ni siquiera está interesado en dejar de hacer las cosas como habitualmente las ha realizado.

"Lo que pasa es que son tan pocos que uno se entrena para manejar una situación de estas y después no se da más" (I.D.1, 2016).

"En cuanto el tiempo lo permita y las actividades de uno como docente, ya que no hay tiempos adicionales para eso" (E.D.1, 2016).

Llama la atención que ambos testimonios son de docentes activos en las instituciones que jalonan el tema de la educación inclusiva y los entornos accesibles, lo que resulta paradójico. Por último, en la gran mayoría de ellos, se reconoce la necesidad sentida y la disposición para recibir formación que les permita desarrollar las competencias para la docencia con la población en condición de diversidad funcional, en tanto es uno de los generadores de ansiedad significativa frente a su rol.

Asimismo, puede darse cuenta que la mayoría de los docentes en estos casos se la juega con sus propios recursos para acompañar al estudiante en esta condición. Frente a la pregunta ¿estaría dispuesto a recibir capacitación para el acompañamiento de la población con diversidad funcional?, los docentes respondieron:

"Me parece muy necesaria, porque como docentes tenemos la responsabilidad de formarnos en asuntos que tengan que ver en términos generales con la educación y este es un tema que tiene que ver y cada vez estamos recibiendo más personas con algún tipo de discapacidad o de diversidad funcional" (D.D.1, 2016).

"Considero que es una gran responsabilidad, pero a la vez es un reto y una oportunidad, para poner a prueba nuestros conocimientos y la aplicación de estrategias funcionales de aprendizaje para esta población" (H.D.1, 2016).

En cuanto al reconocimiento de la política institucional es imprescindible acercar a la población docente a las directrices institucionales para el acompañamiento de los estudiantes con diversidad funcional. Para ello se requiere generar en primera instancia sensibilización frente a las necesidades de la población; en segundo lugar, capacitación en ajustes metodológicos, curriculares, tecnológicos y por último dedicación de tiempos.

La realidad es que la gran mayoría desconoce los lineamientos o no dan cuenta de su existencia, como puede evidenciarse en el testimonio del informante:

"No la conozco, pero sí sé que existe y he visto estudiantes con discapacidad por ahí" (C.D.1, 2016).

"Institucionalmente no existe, pero sí, sé de todas las normativas que hay a nivel de la legislación, deberían tenerse en cuenta para poderse adaptar a la institución. Yo creo que 
se está trabajando al respecto, pero creo que hay mucho por hacer" (D.D.1, 2016).

En las instituciones anteriores, si bien no hay una política instituida como tal, se registra un avance en el tema; sin embargo, el reconocimiento se queda en la población con diversidad escasamente, en tanto no se hace una difusión que comprometa al docente a la aproximación con las estrategias.

\section{Educación inclusiva, un reto a alcanzar}

Con respecto a los entornos educativos para la población con diversidad funcional, "normalmente las aulas no disponen de los recursos necesarios para favorecer la inclusión de estos" (Castellana y Bars, 2006, p. 210).Una de las informantes (E9) con baja visión comentó que una de las cosas que más la impactó fue "la iluminación en el tema de la infraestructura, en algunos de los salones" (2017).Otro estudiante con diversidad funcional visual (E5), respecto a la movilidad al interior de la IES cuando se dirige a clases, expresó: "voy bien hasta que la guía se parte, y yo me pierdo, yo me desubico completamente y sin querer me voy para otros puntos" (2017).

Ainscow (2012) expresa que "conceptualizar la educación inclusiva como un proceso de educación en la diversidad suscita preguntas respecto a qué tipo de apoyo adicional que cada alumno en particular necesita" (p. 44). La posibilidad de implementar en las aulas el material didáctico y tecnológico que se consolide como apoyo para el aprendizaje es bastante distante, ya que requiere de planeación, además de la destinación de partidas presupuestales para ello.

A excepción de dos de las universidades participantes que se conciben como incluyentes en la investigación, solo un par más de instituciones cuentan con algunas máquinas como el Allreader que puede leer textos impresos, para personas con limitaciones visuales parciales o totales, además de la instalación de algunos software para personas ciegas o con baja visión cuya descarga es gratuita, instalados en uno o máximo dos equipos; el resto de las IES presenta mínimos ajustes de tipo locativo.

Los mayores avances que se reconocen en las universidades, son las adecuaciones infraestructurales; sin embargo siguen siendo insuficientes, puesto que responden más a acciones aisladas que pretenden dar solución inmediata al requerimiento por parte de algún estudiante con diversidad funcional motora y no porque sean provenientes de una planeación articulada a los planes de desarrollo institucional, puesto que la mayoría de ellas no da cuenta de una política a partir de lineamientos claros, pero sobre todo que sean de conocimiento público de la comunidad académica.

Retornando al tema de la política pública, se emitió en Colombia el decreto 1421 del 29 de agosto de 2017, el cual, aun desde la autonomía que se ha otorgado a las IES, insta al diseño de los lineamientos para la política institucional que se acoja al marco normativo nacional e internacional. En ella se plantea una terminología a utilizar en lo referente a la diversidad funcional (discapacidad) y además se condiciona a las IES a la aprobación y renovación de procesos como registros calificados de los programas y acreditaciones en calidad, entre otros procesos. Seguramente esta normativa será acatada teniendo en cuenta que su implementación amerita unos tiempos lógicos.

Este decreto además propone una estrategia denominada PIAR ${ }^{1}$ (Plan Individual de Ajustes Razonables), en tanto cada persona es un universo que requiere ajustes diferenciales

\footnotetext{
${ }^{1}$ Herramienta utilizada para garantizar los procesos de enseñanza y aprendizaje de los estudiantes, basados en la valoración pedagógica y social, que incluye los apoyos y ajustes razonables requeridos, entre ellos los curriculares, de infraestructura y todos los demás necesarios para garantizar el aprendizaje, la participación, permanencia y promoción. Son insumo para la planeación de aula del respectivo docente y el Plan de Mejoramiento Institucional (PMI) (pp. 5-6).
} 
en pro de lograr el desarrollo de las competencias técnicas y transversales planteadas desde cada uno de los programas de formación. Enfatiza que las IES deben establecer los lineamientos pero además que "fomenten la incorporación de los lineamientos de política de educación superior inclusiva y motiven la fijación progresiva de su presupuesto para adelantar investigación e implementar estrategias de admisión, evaluación y desarrollo de currículos accesibles, la vinculación y formación de talento humano, el fortalecimiento de los recursos didácticos, pedagógicos y tecnológicos apropiados, garantizando la accesibilidad y permanencia en los programas de educación superior para las personas con discapacidad" (Decreto 1421, 2017,p.19).

El logro de la educación incluyente es un reto que requiere del esfuerzo mancomunado de las partes que conforman el colectivo social. Desde el estado, en la medida que actualice permanentemente la política pública haciéndola cada vez más cercana a las necesidades de las personas con diversidad funcional y velando por su implementación y cumplimiento; desde las IES, garantizando una formación de calidad y equidad; desde la sociedad, mediante la sensibilidad y respeto que se merecen en tanto sujetos dignos, y por último, que la población con diversidad funcional se autodetermine para reclamar y hacer uso pleno de sus derechos, como un aspecto constitutivo de su humanidad.

\section{Discusión}

La presente investigación permite esclarecer algunos asuntos que dan lugar a identificar las generalidades en materia de educación superior inclusiva en Medellín, Colombia; en primera instancia se reconoce que la información respecto al dato de la población con diversidad funcional, no se encuentra actualizada, en tanto el último censo realizado data del año 2005, generando subregistro. Si bien existe el Registro para la Localización y Caracterización de Personas con Discapacidad (RLCPD), como sistema de reporte, muchas de ellas o sus cuidadores no saben de su existencia o no tienen acceso tecnológico a él, tal como lo afirman Cruz y Hernández (2008).

En segundo lugar, con respecto a la población docente, las prácticas pedagógicas al interior de las aulas deben ser pensadas por el docente universitario como estrategias que permitan la disolución de las barreras, en primera instancia de las sociales, que suelen ser sin duda alguna las que promueven actitudes discriminatorias, en coincidencia con Granada, Pomés y Sanhueza (2013).

Posibilitar la integración educativa requiere identificar las capacidades y no las discapacidades de las personas con diversidad funcional, garantizando las mismas oportunidades y participación que la de estudiantes convencionales (Palomino, 2011).

Las IES deben poseer las políticas que garanticen la educación inclusiva, los apoyos y ajustes razonables, en materia tecnológica e infraestructural, así como una actualización permanente de estrategias que habiliten al cuerpo docente, para fomentar la educación con calidad y equidad para la población con diversidad funcional tal como lo concluye Hurtado (2016).

El desconocimiento de los lineamientos y las estrategias didácticas por parte de los docentes para acompañar el proceso de formación de los estudiantes con diversidad funcional, es generador de altos niveles de angustia, ya que solo la buena voluntad, que si bien es necesaria, no es suficiente para evitar que sus derechos estén siendo vulnerados e incluso se ponga en riesgo a la persona (Vargas, Ramos, Cristancho y Parra, 2011). Asimismo, los docentes regulares no cuentan con la preparación y las competencias que les permitan ser inclusivos (Azcárraga, Correa, y Henríquez, 2013).

En condiciones generales se gestiona el acceso y permanencia para esta población al interior de las IES, y además se identifican diferentes niveles de acompañamiento que pasan desde adecuaciones locativas simples 
hasta programas estructurados que evidencian una preocupación genuina por la educación inclusiva, estableciendo similitudes con el estudio de Ricardo y Angarita (2014).

Se resaltan los efectos del factor humano y vincular para los estudiantes con diversidad funcional, logrando sobreponer sus barreras mentales en tanto se sienten parte activa de las IES, desarrollando motivación y sentido de pertenencia y también infortunadamente, el impacto que genera el rechazo social y la exclusión en ellos (Escudero y Martínez, 2011).

Teniendo en cuenta la relevancia de la dimensión relacional del ejercicio docente se debe propiciar el desarrollo de competencias para aprovechar dicho potencial con los estudiantes (Laudadío y Mazzitelli, 2018).

Si bien el modelo social es el implementado en el contexto colombiano, apenas se reconocen algunas propuestas incipientes que se reducen a la accesibilidad (espacios físicos) y la suficiencia en la presentación de política pública, se identifica, por el contrario, un modelo salubrista enfocado en el asistencialismo, que reduce la legislación al cumplimiento exiguo y minimiza las capacidades diversas.

La propuesta de una educación inclusiva puede reconocerse con desarrollos destacados en los niveles de primaria y secundaria, someramente en la educación superior, con mayor impacto para las personas con diversidad funcional motora; en segundo lugar, para las visuales y casi inexistente para auditivas, cognitivas y psicológicas, en tanto no son fácilmente visibilizadas. ¿Acaso sus derechos también son invisibilizados? ¿Su condición las exime de una educación de calidad? ¿Puede reconocerse para ellos aún, los efectos del modelo de prescindencia? Al respecto tal vez sean más los interrogantes a responder que las claridades sobre sus condiciones en las IES.

\section{Referencias bibliográficas}

Ainscow, M. (2012). Haciendo que las escuelas sean más inclusivas: lecciones a partir del análisis de la investigación internacional. Revista de Educación Inclusiva, 5(1), 39-49. Recuperado de http://www.revistaeducacioninclusiva.es/index.php/REI/article/view/220

Alarcón, M.S., Lissi, M.R., Medrano, D., y Loret, H. (2013). La inclusión en la educación superior: desde la voz de estudiantes chilenos con discapacidad. Revista Iberoamericana de Educación, 63, 77-98. Recuperado de https:// rieoei.org/RIE/article/view/502

Azcárraga, M.G., Correa, M.P.P. y Henríquez, S.S. (2013). Actitud de los profesores hacia la inclusión educativa. Papeles de trabajo, Centro de Estudios Interdisciplinarios en Etnolingüística y Antropología Socio-Cultural, 52, 51-59. Recuperado de http://biblioteca.puntoedu. edu.ar/bitstream/handle/2133/3301/n25a03. pdf? sequence $=1 \&$ is Allowed $=y$

Castellana, M.y Bars, I.S. (2006). La inclusión de los estudiantes con discapacidad en la universidad: un reto para la universidad española en el nuevo espacio europeo de la educación superior. Aloma: Revista de Psicología, Ciències de l'educació $i$ de l'esport Blanquerna, (18), 209-227. Recuperado de http://www.raco.cat/ index.php/Aloma/article/view/100812.

Chiny Naranjo, J., Salas Pérez, K.V. y Vargas Dengo, M.C. (2008). Accesibilidad para ingresar a la educación superior: desafíos y logros desde el enfoque de la diversidad. Revista Electrónica Educare, 12(1), 71-82.Recuperado de http://www.revistas.una. ac.cr/index.php/EDUCARE/article/view/1415.

Cruz-Velandia, I., y Hernández-Jaramillo, J. (2008). Magnitud de la discapacidad en Colombia: una aproximación a sus determinantes.Revista Ciencias de la Salud, 6(3), 23-35. Recuperado dehttp://www. scielo.org.co/scielo.php?script=sci_abstract\&pid=S1692-72732008000300003

De Educación, L.G. (1994). Ley 115 febrero 8 de 1994. Constitución Política Colombiana. Ediciones Populares. Recuperado de http://www.mineducacion.gov.co/1621/articles-85906_archivo_pdf.pdf.

Del Carmen Orozco, E. (2009). Las teorías asociacionistas y cognitivas del aprendizaje: diferen- 
cias, semejanzas y puntos en común. Repositorio Universidad de Castilla-La Mancha (UCLM). Recuperado de https://ruidera.uclm. es/xmlui/handle/10578/8200

Departamento Administrativo Nacional de Estadística (DANE) (2015). Sala situacional de personas con discapacidad 2015. Recuperado de http://discapacidadcolombia.com/index. php/estadisticas/185-estadisticas-2015

Escudero, J.M. y Martínez, B. (2011). Educación inclusiva y cambio escolar. Revista Iberoamericana de Educación, 55, 85-105. Recuperado de https://s3.amazonaws.com/ academia.edu.documents/33988041/inclusion. pdf?AWSAccessKeyId=AKIAIWOWYYGZ2Y53UL3A\&Expires $=1527541409 \&$ Signature $=$ LeJiyz9iUsctKbvnsDhNZfaY6Cc\%3D\&response-content-disposition=inline $\% 3 \mathrm{~B} \%$ 20filename\%3DInclusion.pdf

Fernández de Álava, M., Barrera Corominas, A., y Díaz Vicario, A. (2013). La inclusión en instituciones iberoamericanas de educación superior. Revista Iberoamericana de Educación, (63), 99-113. Recuperado de https://ddd. uab.cat/record/123400

Fernández, F.H. y Duarte, J.E. (2016). Retos de la inclusión académica de personas con discapacidad en una universidad pública colombiana. Formación Universitaria,9(4), 95-104. Recuperado de https://scielo.conicyt.cl/pdf/ formuniv/v9n4/art11.pdf. doi: 10.4067/S071850062016000400011

Galeano, M.E. (2003). Diseño de proyectos de investigación cualitativa. Medellín: Fondo Editorial Universidad Eafit.

Granada Azcárraga, M., Pomés Correa, M.P. y Sanhueza Henríquez, S. (2013). Actitud de los profesores hacia la inclusión educativa. Recuperado de http://biblioteca.puntoedu. edu.ar/bitstream/handle/2133/3301/ n25a03.pdf? sequence $=1$ \&isAllowed $=y$

Hernández Sampieri, R., Fernández Collado, C. y Baptista Lucio, P. (2010). Metodología de la investigación. Recuperado de https:// trabajosocialudocpno.files.wordpress. com/2017/07/metodologc3a3c2ada_de la_investigacic3a3c2b3n_-sampieri-_6ta edicion1.pdf

Hurtado, L.T. y Ágüelo, M.A. (2014). Inclusión educativa de las personas con discapacidad en Colombia. Revista Ces, Movimiento y Salud, 2(1), 45-55.Recuperado de http://revistas.ces.edu.co/index.php/movimientoysalud/article/view/2971

Laudadío, J. y Mazzitelli, C. (2018). Adaptación y validación del Cuestionario de Relación Docente en el Nivel Superior. Interdisciplinaria, 35(1). Recuperado de http:// www.ciipme-conicet.gov.ar/ojs/index.php/ interdisciplinaria/article/view/456/67

Martínez-Salgado, C. (2012). El muestreo en investigación cualitativa. Principios básicos y algunas controversias. Revista Ciência \& Saúde Coletiva, 17(3). Recuperado de https://www.scielosp.org/article/ ssm/content/raw/?resource_ssm_path=/ media/assets/csc/v17n3/v17n3a06.pdf. doi: 10.1590/S1413-81232012000300006

OMS. (2011) Informe mundial sobre la discapacidad. Recuperado de http://www.who. int/disabilities/world_report/2011/accessible_es.pdf

ONU. (2006) Convención Internacional sobre los Derechos de las Personas con Discapacidad. Recuperado dehttp://www.un.org/ spanish/disabilities/default.asp?id=497

Palacios, A., y Romañach, J. (2008). El modelo de diversidad: una nueva visión de la bioética desde la perspectiva de las personas con diversidad funcional (discapacidad). Intersticios, 2(2), 37-47. Recuperado de http://www.intersticios.es/article/ view/2712

Palomino, A.S. (2011). La Universidad de Almería ante la integración educativa y social de los estudiantes con discapacidad: Ideas y actitudes del personal docente e investigador Revista de Educación, 354, 575-603. Recuperado de http://www.revistaeducacion.educacion.es/re354/re354_23. pdf

Papalia, D., Wendkos, S., y Duskin, R. (2001). Psicología del desarrollo. Colombia: Mc Graw Hill. 
Ricardo, T.D. (2012). Acceso y movilización de personas con discapacidad físico-motriz en instituciones de educación superior Cartagena de Indias. Saber Ciencia y Libertad, 7(2), 39-51. Recuperado de http://ojs.sabercienciaylibertad.com/ojs/ index.php/scyl/article/view/125

Ricardo, A.C. y Angarita, M.M. (2014). Educación superior y discapacidad: análisis desde la experiencia de algunas universidades colombianas. Revista Española de Discapacidad, 2(2), 83-101. Recuperado de http://www.cedd.net/redis/index.php/ redis/article/view/130/pdf_15

Rubio, A.V. (2017). Inclusión de Personas en Situación de Discapacidad en Educación Superior, desde el Enfoque de la Responsabilidad Social, en un Contexto de Transiciones Discursivas respecto del Binomio Integración/Inclusión. Revista latinoamericana de educación inclusiva, 11(2), 199-216. Recuperado dehttps://scielo. conicyt.cl/pdf/rlei/v11n2/art13.pdf. doi: 10.4067/S0718-73782017000200013

Taylor, S.J. y Bogdan, R. (1992). Introducción a los métodos cualitativos en investigación. La búsqueda de los significados. España: Paidós.
Vargas, M.C., Ramos, M.A., Cristancho, C.A. y Parra, L.M. (2011). La universidad Manuela Beltrán, evolucionando hacia una universidad más inclusiva. Umbral Cientifico, (18). Recuperado de http://www. redalyc.org/html/304/30421523006/

Velásquez. R. (2009). Hacia una nueva definición del concepto "política pública". Desafios, 20(1). Recuperado de http://www. redalyc.org/pdf/3596/359633165006.pdf

Valenzuela-Zambrano, B., Panão Ramalho, A., Chacón-López, H. y López-Justicia, M.D. (2017). Alumnado con discapacidad en Educación Superior en Chile y Portugal: Una revisión de las políticas y prácticas que fomentan la cultura inclusiva. Recuperado de: http://digibug.ugr. es/bitstream/handle/10481/45790/03\%20

-\%20Barbara\%20Valenzuela-Zambrano $\% 3 \mathrm{~b} \% 20$ Anabela $\% 20$ Panao $\% 20$ Ramalho\%3b\%20Helena\%20Chacon-Lopez\%3b\%20Maria\%20Dolores\%20LopezJusticia.pdf? sequence $=1$ \&isAllowed $=\mathrm{y}$

World Health Organization. (2001). Clasificación internacional del funcionamiento de la discapacidad y de la salud: CIF. Recuperado de http://apps.who.int/iris/bitstream/10665/42419/1/9243545426.pdf

Recibido: 27 de noviembre de 2017 Aceptado: 23 de septiembre de 2019 